\title{
Prediction of rapid intensification of tropical cyclones with deep learning
}

This manuscript has been submitted for publication. The current version is a nonpeer reviewed preprint submitted to EarthArXiv.

\section{Authors and Affiliation}

Sambatra Andrianomena ${ }^{1,2}$, Mika Rafieferantsoa ${ }^{3, *}$, Holifidy Rapanoël ${ }^{3}$, and Rondrotiana Barimalala ${ }^{4}$

${ }^{1}$ South African Radio Astronomy Observatory (SARAO), Black River Park, Observatory, Cape Town, 7925, South Africa.

${ }^{2}$ University of the Western Cape, Bellville, Cape Town 7535, South Africa.

${ }^{3}$ A\&A, Department of Physics, Faculty of Sciences, University of Antananarivo, B.P. 906, Antananarivo 101, Madagascar

${ }^{4}$ University of Cape Town, Rondebosch, Cape Town 7701, South Africa.

*Corresponding author: rafieferantsoamika@gmail.com 





other study ${ }^{16}$. It results from the combination of a residual network $\left(\operatorname{ResNet}^{17}\right)$ with inception modules ${ }^{18}$. In order to decrease the error, which deeper architectures are more prone to, the use of residual layers is proposed to improve the performance ${ }^{17}$. To learn from different scales at higher levels of the network, two inception modules are added after the last residual layer. The third architecture is the Xception model which inherits from the Inception structure where the modules are substituted with depth-wise separable convolutions ${ }^{10}$. That replacement made Xception outperform the Inception architecture on Imagenet data due to a more efficient use of the model parameters. We also exploit the capacity of Xception network in this study. The only modifications that have been implemented in the original architecture are the adaptation of the input layer to expect a 1 channel input, instead of 3, and the addition of a dense layer with one unit at the end of the chain to reflect our binary classification problem.

\section{Performance measure}

For easy reference, the four entries of a confusion matrix in a binary classification is defined as follows $\left[\begin{array}{ll}T N & F P \\ F N & T P\end{array}\right]$. Where, True Positive (TP) is the number of positive instances that are properly classified, True Negative (TN) is the number of negative instances that are well identified, False Positive (FN) is the number of negative instances that are misclassified as positive ones and False Negative (FN) is the number of positive examples that are identified as negative ones. To assess the performance of the methods, the following metrics are used

- recall also known as probability of detection $(P O D)$ or sensitivity. It denotes how well the classifier is able to minimize the number of positive instances identified as negative (false negative). It is given by

$$
\text { recall }=\frac{\mathrm{TP}}{\mathrm{TP}+\mathrm{FN}}
$$

- Heidke Skill Score (HSS) which indicates the improvement on a classifier with a random guess and reads

$$
H S S=\frac{\mathrm{TP} \times \mathrm{TN}-\mathrm{FP} \times \mathrm{FN}}{(\mathrm{TP}+\mathrm{FN}) \times(\mathrm{FN}+\mathrm{TN})+(\mathrm{TP}+\mathrm{FP}) \times(\mathrm{TN}+\mathrm{FP})}
$$

- false alarm ratio known as FAR, defined by

$$
F A R=\frac{\mathrm{FP}}{\mathrm{FP}+\mathrm{TP}}
$$

. A low value of this metric indicates a good classifier.

- Receiving Operating Characteristic - Area Under the Curve (roc-auc) is the result of averaging the variation of the true positive rate as a function of false positive rate. It indicates the degree of separability, in other words the capability of the model to distinguish between positive and negative examples.

\section{References}

1. Gray, W. M. Global view of the origin of tropical disturbances and storms. Mon. Weather. Rev. 96, 669-700, DOI: 10.1175/1520-0493(1968)096<0669:GVOTOO>2.0.CO;2 (01 Oct. 1968).

2. Kaplan, J. \& DeMaria, M. Large-scale characteristics of rapidly intensifying tropical cyclones in the north atlantic basin.
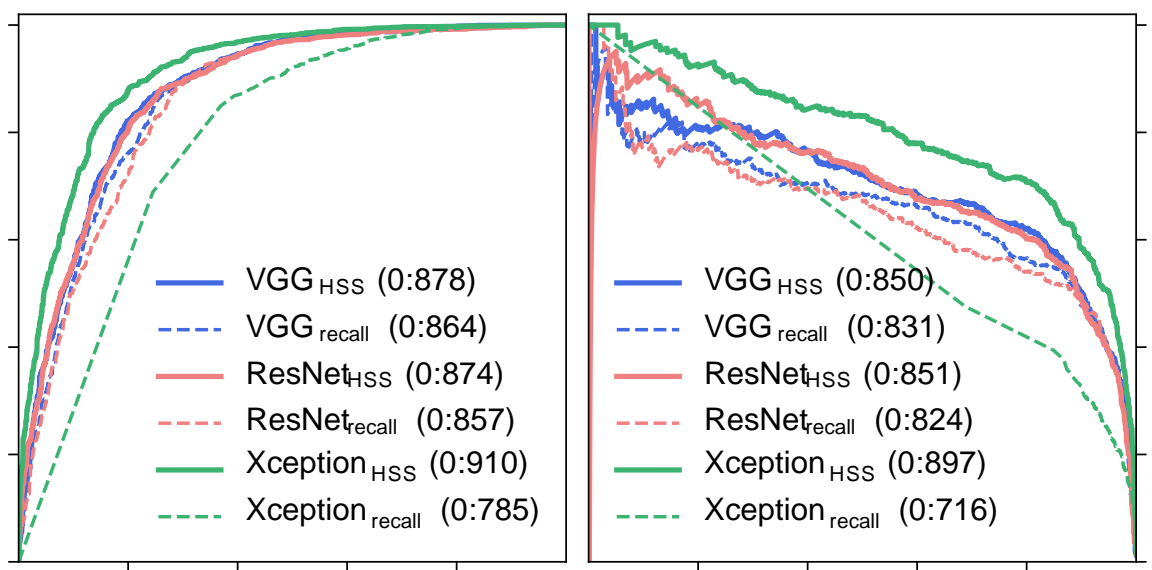

tropical cyclones in the south west ).1002/2016JC012028 (2017). https:

$\mathrm{d}$ intensification of tropical cyclones ather. Rev. 146, 3773 - 3800, DOI:

'physical environment. In: Managing A Special Report of Working Groups I srsity Press, Cambridge, UK, and New 
6. Grimes, A. \& Mercer, A. E. Synoptic-scale precursors to tropical cyclone rapid intensification in the atlantic basin. Adv. Meteorol. 2015, 814043, DOI: 10.1155/2015/814043 (2015).

7. Mercer, A. \& Grimes, A. Diagnosing tropical cyclone rapid intensification using kernel methods and reanalysis datasets. Procedia Comput. Sci. 61, 422-427, DOI: https://doi.org/10.1016/j.procs.2015.09.179 (2015). Complex Adaptive Systems San Jose, CA November 2-4, 2015.

8. Mercer, A. \& Grimes, A. Atlantic tropical cyclone rapid intensification probabilistic forecasts from an ensemble of machine learning methods. Procedia Comput. Sci. 114, 333-340, DOI: https://doi.org/10.1016/j.procs.2017.09.036 (2017). Complex Adaptive Systems Conference with Theme: Engineering Cyber Physical Systems, CAS October 30 - November 1, 2017, Chicago, Illinois, USA.

9. $\mathrm{Su}, \mathrm{H}$. et al. Applying satellite observations of tropical cyclone internal structures to rapid intensification forecast with machine learning. Geophys. Res. Lett. 47, e2020GL089102, DOI: https://doi.org/10.1029/2020GL089102 (2020). https://agupubs.onlinelibrary.wiley.com/doi/pdf/10.1029/2020GL089102.

10. Chollet, F. Xception: Deep learning with depthwise separable convolutions. CoRR abs/1610.02357 (2016). 1610.02357.

11. Knapp, K. R. \& Kossin, J. P. New global tropical cyclone data set from ISCCP B1 geostationary satellite observations. $J$. Appl. Remote. Sens. 1 - 6 (2007).

12. Shaiba, H. \& Hahsler, M. Applying machine learning methods for predicting tropical cyclone rapid intensification events. Res. J. Appl. Sci. Eng. Technol. 13, 638-651 (2016).

13. Kossin, J. P., Knapp, K. R., Vimont, D. J., Murnane, R. J. \& Harper, B. A. A globally consistent reanalysis of hurricane variability and trends. Geophys. Res. Lett. 34, DOI: https://doi.org/10.1029/2006GL028836 (2007). https://agupubs. onlinelibrary.wiley.com/doi/pdf/10.1029/2006GL028836.

14. Knapp, K. R. Calibration assessment of isccp geostationary infrared observations using hirs. J. Atmospheric Ocean. Technol. 25, 183 - 195, DOI: 10.1175/2007JTECHA910.1 (01 Feb. 2008).

15. Simonyan, K. \& Zisserman, A. Very deep convolutional networks for large-scale image recognition. arXiv preprint arXiv:1409.1556 (2014).

16. Hassan, S., Andrianomena, S. \& Doughty, C. Constraining the astrophysics and cosmology from $21 \mathrm{~cm}$ tomography using deep learning with the ska. Mon. Notices Royal Astron. Soc. 494, 5761-5774 (2020).

17. He, K., Zhang, X., Ren, S. \& Sun, J. Deep residual learning for image recognition. In Proceedings of the IEEE conference on computer vision and pattern recognition, 770-778 (2016).

18. Szegedy, C. et al. Going deeper with convolutions. In Proceedings of the IEEE conference on computer vision and pattern recognition, 1-9 (2015).

\section{Acknowledgements}

S.A., M.R., H.R. and R.B. are thankful to Microsoft Azure for providing computing resources and technical supports. S.A. acknowledges support of the South African Radio Astronomy Observatory. R.B. was supported by the Royal Society FLAIR Program.

\section{Author contributions statement}

All authors contributed to the conception of the work, discussion of the results and the writing of the manuscript. S.A., M.R. worked on the network architectures and led the writing of the paper, H.R. worked on a code that was used for labeling the raw data. R.B. contributed on the climate science part of the work.

\section{Additional information}

\section{Data availability}

HURSAT-B1 data are freely available at https://www.ncei.noaa.gov/data/hurricane-satellite-hursat-b1/

archive/v06/

\section{Competing interests}

Authors declare no competing interests. Correspondence and requests for materials should be addressed to M.R. (rafieferantsoamika@gmail.com). Reprints and permissions information is available at www.nature.com/reprints. 